\title{
PROGRAMMING AND TESTING OF A FRIENDLY GUI-MATLAB SOFTWARE TO PREDICT THE THERMODYNAMIC PROPERTIES OF WATER
}

\author{
P.F. Arce-Castillo ${ }^{1}$, N.V. Freire ${ }^{2}$ \\ ${ }^{1}$ Chemical Engineering Department, Engineering School of Lorena, University of São Paulo, Lorena, Brazil \\ ${ }^{2}$ Chemical Engineering Department, Engineering School of Lorena, University of São Paulo, Lorena, Brazil
}

\begin{abstract}
Water is the most used fluid in industrial processes, such as heating, cooling, etc. This work aims the creation of a program in MatLab software with an interface in GUI to correlate and calculate the thermodynamic properties of this fluid, such as enthalpy, entropy, internal energy and specific volume, under many routines and with a great working range of pressure and temperature. This program provides good results, showing that it can be used to design equipments in industrial processes and to support the teaching disciplines of Applied Thermodynamic. Presentation is made friendly through of Graphical Unit Interfaces (GUIMatLab).
\end{abstract}

Keywords: Thermodynamic properties, Water, GUI-MatLab

\section{INTRODUCTION}

Today, the water is the most used fluid, present in a great variety of industrial processes, such as heating and cooling, distillation, generation of mechanical work in steam power cycles, etc[1-4]. In these processes, the conditions of pressure and temperature varies, which causes state changes in the fluid and a variation in its thermodynamic properties, such as enthalpy, entropy, specific volume, internal energy, quality and exergy[5].

With the growth of computing and the need to develop economically viable processes with a large production capacity, programs to calculate the properties of the substances became very useful, providing accurate results quickly and allowing the process optimization. Besides, with the development of new materials and the enhancement of technology, these programs need to have a large working range to be possible the calculation of properties at near and above the critical conditions. Thus, a program to calculate the thermodynamic properties of water, the most used fluid in industry, in a wide working range is an excellent tool in a great number of industrial plants, mainly in processes with heat exchanges, present in any chemical industry, and steam power cycles, such as Rankine cycle, one of the methods most used for the generation of electricity in thermoelectric power plants in the world [6].

In the Internet, there are many available programs to this purpose, but they don't work in a working range so large, rarely operating above the critical point of water. But, today, with the development of new and more efficient technologies, results located at severe thermodynamic conditions become indispensable to design processes more complex. Thus, this work aims to create a program in MatLab software to predict the main thermodynamic properties of water in four different thermodynamic states (compressed liquid, saturated mixture, superheated vaporand supercritical fluid) in a large working range to pressure and temperature. The program created has an interface in GUI to allow a good interaction with the user.

The program code uses values provide by the user to begin the calculation of the thermodynamic properties of water. The user needs to provide, at least, two conditions of the system to determinate other properties.

With these known properties of water, it is possible to calculate the generated work and efficiency of a power cycle which uses water, the heat exchange in processes of heating and cooling, among others $[7,8]$. These values can be used to simulate the processes and design equipments, showing the great range of applications to the program. Besides, the program can also be usedfor teaching courses of Applied Thermodynamics in universities.

\section{METHODOLOGY}

There are many ways to predict and calculate the thermodynamic properties of a fluid. This determination may be made by equations of state or thermodynamic tables [9]. In both cases, however, it is necessary, at least, the knowledge of two thermodynamic properties of the system to begin the calculation and determinate other thermodynamic properties. Based on this, the program, created in MatLab with an interface (GUI) has eleven options to predict the thermodynamic properties of the water. These options, with their specific units, are:

1. Pressure (bar) and Temperature $\left({ }^{\circ} \mathrm{C}\right)$;

2. Pressure (bar) and Enthalpy $(\mathrm{kJ} / \mathrm{kg})$;

3. Pressure (bar) and Entropy (kJ/kg.K);

4. Pressure (bar) and Specific Volume $\left(\mathrm{m}^{3} / \mathrm{kg}\right)$; 
5. Pressure (bar) and Internal Energy $(\mathrm{kJ} / \mathrm{kg})$;

6. Pressure (bar) and Quality;

7. Temperature $\left({ }^{\circ} \mathrm{C}\right)$ and Enthalpy $(\mathrm{kJ} / \mathrm{kg})$;

8. Temperature $\left({ }^{\circ} \mathrm{C}\right)$ and Entropy $(\mathrm{kJ} / \mathrm{kg} . \mathrm{K})$;

9. Temperature $\left({ }^{\circ} \mathrm{C}\right)$ and Specific Volume $\left(\mathrm{m}^{3} / \mathrm{kg}\right)$;

10. Temperature $\left({ }^{\circ} \mathrm{C}\right)$ and Internal Energy $(\mathrm{kJ} / \mathrm{kg})$;

11. Temperature $\left({ }^{\circ} \mathrm{C}\right)$ and Quality.

The user should choose the option and supply to the program the required properties values. The program, after receive the values of the properties, will calculate, with the internal correlations inside of its code and using spline methods, other thermodynamic properties, in addition to the exergy and the determination of the thermodynamic state where the system is located.
The program operates with pressure range from 0,0061 bar until 1000,0 bar and temperature range from $0,01^{\circ} \mathrm{C}$ until $1300,0^{\circ} \mathrm{C}$. The working range of other thermodynamic properties is determined based on internal correlations of the program.

Furthermore, the program code comprises four thermodynamic states of the water. These states are:

- Superheated Vapor;

- Compressed Liquid;

- $\quad$ Saturated Mixture;

- Supercritical Fluid.

In the interface developed with GUI-MatLab, there is a figure that represents the phase diagram of the water, which facilitates the user understanding about the fluid phase behavior. This interface is showed in Fig. 1.

Thermodynamic Properties of Water
Chemical Engineering Departiment, EEL - USP
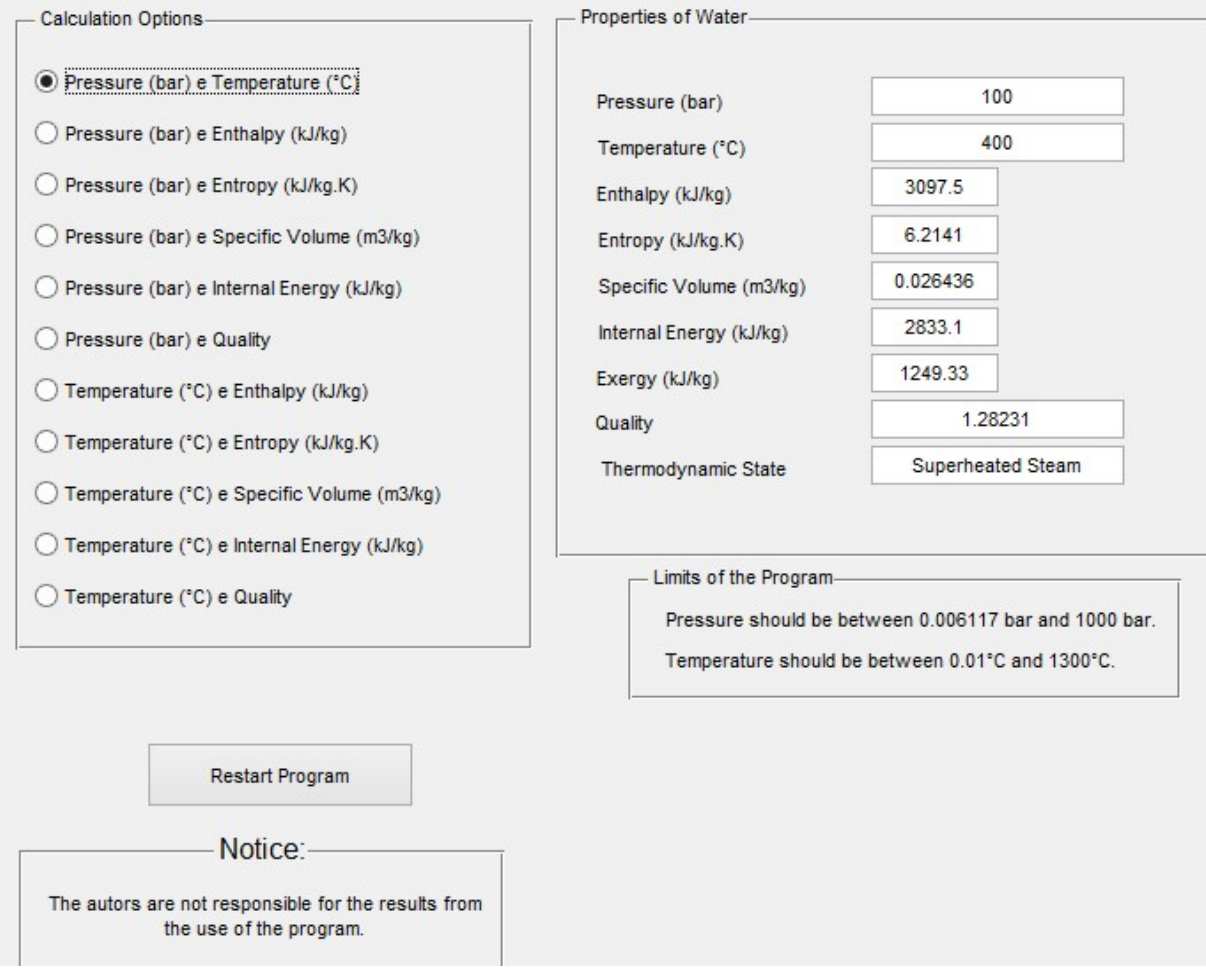

Fig -1: Interface in GUI-MatLab for predicting the thermodynamic properties of water.

\section{RESULTS ANS DISCUSSIONS}

Results obtained with the program developed in this work were compared with the others ones obtained with others programs that also calculate the thermodynamic properties of water.
One of them is PropVap [10]. This program has four options to predict the thermo dynamic properties of water and operates from the triple point of water until 190,0 bar and $1300,0^{\circ} \mathrm{C}$

A second program used is CATT 3 [11]. This program has more options than PropVap and its working range is bigger than PropVap too. 
A third program used is XSteam, a program developed in MatLab, that has options similar to PropVap, but operates in a larger working range for the pressure. It is important to mention that none of the programs used to compare the results have the option of calculating exergy or do not have the eleven options presented in this work.

Results obtained with these three programs and the results obtained with the computational program developed in this work are shown in Tables 1, 2,3 and 4 for the four thermodynamic states: Superheated steam, saturation, compressed liquid and supercritical regions, respectively.

In Tables 1, 2, 3 and 4, $\mathrm{P}$ represents the pressure (bar), $\mathrm{T}$ is the temperature $\left({ }^{\circ} \mathrm{C}\right), \mathrm{H}$ is the enthalpy $(\mathrm{kJ} / \mathrm{kg}), \mathrm{U}$ is the internal energy $(\mathrm{kJ} / \mathrm{kg}), \mathrm{S}$ is the entropy $\left(\mathrm{kJ} / \mathrm{kg} .{ }^{\circ} \mathrm{C}\right), \mathrm{Ex}$ is the Exergy $(\mathrm{kJ} / \mathrm{kg}), \mathrm{v}$ is the specific volume $\left(\mathrm{m}^{3} / \mathrm{kg}\right)$ and $\mathrm{x}$ is the quality.

Table 1 presents the results obtained in this work and the results obtained with the other three programs in the superheated vapor region. Given two thermodynamic properties, that is: $\mathrm{P}$ and $\mathrm{T}, \mathrm{P}$ and $\mathrm{H}, \mathrm{P}$, and $\mathrm{S}, \mathrm{P}$ and $\mathrm{v}, \mathrm{P}$ and $\mathrm{U}, \mathrm{T}$ and $\mathrm{H}, \mathrm{T}$ and $\mathrm{S}, \mathrm{T}$ and $\mathrm{V}$ and $\mathrm{T}$ and $\mathrm{U}$, the other thermodynamic properties are calculated. In this table, some thermodynamic properties have not been implemented for the option chosen $(*)$ and many results were not found for the programs because the chosen options were not implemented $(* *)$.

Comparing the results, especially when one of the options contain the pressure, the results for the thermodynamic properties seem to be very similar. The same is true for the case when the temperature is one of the thermodynamic properties of input. Thus, the results obtained in this work and CATT 3 are not different.

Table 2 presents the results obtained in this work and the results obtained with the other three programs in the saturation region. In this work, it is also possible to predict the saturated liquid line $(x=0)$ and the saturated vapor line $(\mathrm{x}=1)$. Given two thermodynamic properties, that is: $\mathrm{P}$ and $\mathrm{H}, \mathrm{P}$, and $\mathrm{S}, \mathrm{P}$ and $\mathrm{v}, \mathrm{P}$ and $\mathrm{U}, \mathrm{P}$ and $\mathrm{x}, \mathrm{T}$ and $\mathrm{H}, \mathrm{T}$ and $\mathrm{S}, \mathrm{T}$ and $\mathrm{v}, \mathrm{T}$ and $\mathrm{U}$ and $\mathrm{T}$ and $\mathrm{x}$, the other thermodynamic properties are calculated. In this table, some thermodynamic properties have not been implemented for the option chosen $\left(^{*}\right)$ and many results were not found for the programs because the chosen options were not implemented $(* *)$.

When the comparison is done between the results obtained in this work with the others ones, especially when the option contains the pressure, the results for the thermodynamic properties seem also to be very similar. The same can be said when the temperature is included in one of the options chosen by the user. For the case of $\mathrm{T}$ and $\mathrm{S}$ and $\mathrm{T}$ and $\mathrm{v}$, the results obtained in this work and CATT 3 are very similar. For the case of $\mathrm{T}$ and $\mathrm{x}$, the results obtained in this work, CATT 3, and Xsteam are highly similar.

In Table 3, results obtained in this work and the results obtained with the other three programs in the compressed liquid region are shown. Given two thermodynamic properties, that is: $\mathrm{P}$ and $\mathrm{T}, \mathrm{P}$ and $\mathrm{H}, \mathrm{P}$, and $\mathrm{S}, \mathrm{P}$ and $\mathrm{v}, \mathrm{P}$ and $\mathrm{U}, \mathrm{T}$ and $\mathrm{S}, \mathrm{T}$ and $\mathrm{V}$ and $\mathrm{T}$ and $\mathrm{U}$, the other thermodynamic properties are calculated. In this table, some thermodynamic properties have not been implemented for the option chosen $\left(^{*}\right)$ and many results were not found for the programs because the chosen options were not implemented $(* *)$.

Table - 1 : Results for the Superheated Vapor region

\begin{tabular}{|c|c|c|c|c|c|c|c|c|}
\hline \multicolumn{9}{|c|}{ Pressure and Temperature } \\
\hline Program & $\mathbf{P}$ & $T$ & $\mathbf{H}$ & $\mathrm{s}$ & $\mathbf{v}$ & $\mathrm{U}$ & $\mathbf{E x}$ & $\mathbf{x}$ \\
\hline This work & \multirow{4}{*}{60,0} & \multirow{4}{*}{400,0} & 3178,30 & 6,5430 & 0,0474 & 2893,70 & 1232,01 & 1,2506 \\
\hline CATT 3 & & & 3177,00 & 6,5410 & 0,0474 & 2893,00 & $\left({ }^{*}\right)$ & $(*)$ \\
\hline PropVap & & & 3177,20 & 6,5407 & 0,0474 & 2892,80 & 1231,60 & 1,2500 \\
\hline XSteam & & & 3178,20 & 6,5400 & 0,0474 & 2893,60 & $\left({ }^{*}\right)$ & $(*)$ \\
\hline
\end{tabular}

\begin{tabular}{|c|c|c|c|c|c|c|c|c|}
\hline \multicolumn{9}{|c|}{ Pressure and Enthalpy } \\
\hline Program & $\mathbf{P}$ & $\mathbf{H}$ & $T$ & $\mathrm{~S}$ & $\mathbf{v}$ & $\mathrm{U}$ & $\mathbf{E x}$ & $\mathbf{x}$ \\
\hline This work & \multirow{4}{*}{100,0} & \multirow{4}{*}{3000,0} & 371,93 & 6,0637 & 0,0242 & 2758,25 & 1196,660 & 1,2083 \\
\hline CATT 3 & & & 370,50 & 6,0650 & 0,0242 & 2758,00 & $\left({ }^{8}\right)$ & $(*)$ \\
\hline PropVap & & & 370,49 & 6,0653 & 0,0242 & 2758,20 & 1196,200 & 1,2090 \\
\hline XSteam & & & 370,34 & 6,0659 & 0,0242 & 2758,10 & $\left({ }^{*}\right)$ & $(*)$ \\
\hline
\end{tabular}

\begin{tabular}{|c|c|c|c|c|c|c|c|c|}
\hline \multicolumn{9}{|c|}{ Pressure and Entropy } \\
\hline Program & $\mathbf{P}$ & $\mathrm{S}$ & $\mathbf{T}$ & $\mathbf{H}$ & $\mathbf{V}$ & $\mathrm{U}$ & Ex & $x$ \\
\hline This work & \multirow{4}{*}{210,0} & \multirow{4}{*}{7,0} & 788,25 & 4032,40 & 0,0224 & 3562,17 & 1949,910 & 4,9459 \\
\hline CATT 3 & & & 788,30 & 4035,00 & 0,0224 & 3565,00 & $\left({ }^{*}\right)$ & $(*)$ \\
\hline PropVap & & & $\left({ }^{* 8}\right)$ & $\left({ }^{* *}\right)$ & $\left({ }^{* *}\right)$ & $\left({ }^{* *}\right)$ & $(* *)$ & $\left({ }^{* *}\right)$ \\
\hline XSteam & & & 788,77 & 4033,90 & 0,0224 & 3563,40 & $\left({ }^{*}\right)$ & $(*)$ \\
\hline
\end{tabular}

\begin{tabular}{|c|c|c|c|c|c|c|c|c|}
\hline \multicolumn{9}{|c|}{ Pressure and Specific Volume } \\
\hline Program & $\mathbf{P}$ & $\mathbf{v}$ & $\mathrm{T}$ & $\mathbf{H}$ & $\mathrm{S}$ & $\mathrm{U}$ & Ex & $\mathbf{x}$ \\
\hline This work & \multirow{4}{*}{150,0} & \multirow{4}{*}{0,03} & 738,88 & 3937,36 & 7,0564 & 3487,34 & 1838,06 & 2,3259 \\
\hline CATT 3 & & & 739,30 & 3940,00 & 7,0570 & 3490,00 & $\left({ }^{*}\right)$ & $(*)$ \\
\hline PropVap & & & $\left({ }^{* *}\right)$ & $\left({ }^{* *}\right)$ & $\left({ }^{* *}\right)$ & $\left({ }^{* *}\right)$ & $\left({ }^{* *}\right)$ & $\left({ }^{* *}\right)$ \\
\hline XSteam & & & $(* *)$ & $(* *)$ & $\left({ }^{* *}\right)$ & $(* *)$ & $(* *)$ & $\left({ }^{* *}\right)$ \\
\hline
\end{tabular}

\begin{tabular}{|c|c|c|c|c|c|c|c|c|}
\hline \multicolumn{9}{|c|}{ Pressure and Internal Energy } \\
\hline Program & $\mathbf{P}$ & $\mathrm{U}$ & $\mathbf{T}$ & $\mathbf{H}$ & $\mathbf{S}$ & $\mathbf{v}$ & $\mathbf{E x}$ & $\mathbf{x}$ \\
\hline This work & \multirow{4}{*}{200,0} & \multirow{4}{*}{3000,00} & 522,19 & 3316,30 & 6,2367 & 0,0157 & 1461,39 & 2,5443 \\
\hline CATT 3 & & & $(* *)$ & $(* 8)$ & $\left({ }^{* *}\right)$ & $(* *)$ & $(* *)$ & $\left({ }^{* *}\right)$ \\
\hline PropVap & & & $(* *)$ & $(* *)$ & $\left({ }^{* *}\right)$ & $(* *)$ & $(* *)$ & $\left({ }^{* *}\right)$ \\
\hline XSteam & & & $(* 8)$ & $(* 8)$ & $\left({ }^{* *}\right)$ & $(* *)$ & $(* *)$ & $\left({ }^{* *}\right)$ \\
\hline
\end{tabular}

\begin{tabular}{|c|c|c|c|c|c|c|c|c|}
\hline \multicolumn{9}{|c|}{ Temperature and Enthalpy } \\
\hline Program & $T$ & $\mathbf{H}$ & $\mathbf{P}$ & $\mathrm{S}$ & $\mathbf{v}$ & $\mathbf{U}$ & $\mathbf{E x}$ & $x$ \\
\hline This work & \multirow{4}{*}{400,0} & \multirow{4}{*}{3000,00} & 140,92 & 5,9394 & 0,0171 & 2759,29 & 1233,73 & 1,3436 \\
\hline CATT 3 & & & $(* *)$ & $(* *)$ & $(* *)$ & $(* *)$ & $(* *)$ & $\left({ }^{* *}\right)$ \\
\hline PropVap & & & $(* *)$ & $(* *)$ & $(* *)$ & $\left({ }^{* *}\right)$ & $(* *)$ & $\left({ }^{* *}\right)$ \\
\hline XSteam & & & $\left({ }^{* *}\right)$ & $(* *)$ & $\left({ }^{* *}\right)$ & $(* *)$ & $\left({ }^{* *}\right)$ & $\left({ }^{* *}\right)$ \\
\hline
\end{tabular}




\begin{tabular}{|c|c|c|c|c|c|c|c|c|}
\hline \multicolumn{9}{|c|}{ Temperature and Entropy } \\
\hline Program & $\mathrm{T}$ & $\mathrm{s}$ & $\mathbf{P}$ & $\mathbf{H}$ & $\mathbf{v}$ & $\mathrm{U}$ & Ex & $\mathrm{x}$ \\
\hline This work & \multirow{4}{*}{500,0} & \multirow{4}{*}{9,00} & 0,70 & 3489,10 & 5,0827 & 3132,46 & 810,31 & 1,3634 \\
\hline CATT 3 & & & 0,70 & 3488,00 & 5,1060 & 3132,00 & $\left({ }^{*}\right)$ & $\left({ }^{*}\right)$ \\
\hline PropVap & & & $(* *)$ & $\left({ }^{(* *)}\right)$ & $\left({ }^{(*)}\right)$ & ${ }^{(* *)}$ & $\left({ }^{* *}\right)$ & $\left({ }^{* *}\right)$ \\
\hline XSteam & & & $(* *)$ & $(* *)$ & $\left({ }^{(*}\right)$ & $\left({ }^{* *}\right)$ & $(* *)$ & $\left({ }^{* *}\right)$ \\
\hline
\end{tabular}

\begin{tabular}{|c|c|c|c|c|c|c|c|c|}
\hline \multicolumn{9}{|c|}{ Temperature and Specific Volume } \\
\hline Program & T & $\mathbf{v}$ & $\mathbf{P}$ & $\mathbf{H}$ & $\mathrm{s}$ & $\mathrm{U}$ & Ex & 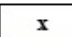 \\
\hline This work & \multirow{4}{*}{400,0} & \multirow{4}{*}{0,02} & 125,14 & 3039,65 & 6,0424 & 2789,34 & 1242,69 & 1,3146 \\
\hline CATT 3 & & & 125,00 & 3039,00 & 6,0410 & 2789,00 & $\left({ }^{*}\right)$ & $(*)$ \\
\hline PropVap & & & $\left({ }^{* *}\right)$ & $\left({ }^{* *}\right)$ & $\left({ }^{(*)}\right)$ & $(* *)$ & $\left({ }^{* *}\right)$ & $(* *)$ \\
\hline XSteam & & & $\left({ }^{(*)}\right)$ & $\left({ }^{* *}\right)$ & $\left({ }^{(*)}\right)$ & $(* *)$ & $(* *)$ & $(* *)$ \\
\hline
\end{tabular}

\begin{tabular}{|c|c|c|c|c|c|c|c|c|}
\hline \multicolumn{9}{|c|}{ Temperature and Internal Energy } \\
\hline Program & $\mathrm{T}$ & $\mathrm{U}$ & $\mathbf{P}$ & H & $\mathrm{s}$ & $\mathbf{v}$ & Ex & $\mathbf{x}$ \\
\hline This work & \multirow{4}{*}{250,0} & \multirow{4}{*}{2700,00} & 13,53 & 2929,73 & 6,7672 & 0,1712 & 916,65 & 1,0722 \\
\hline CATT 3 & & & $(* *)$ & $\left({ }^{* *}\right)$ & $\left({ }^{(* *}\right)$ & $\left({ }^{(* *)}\right.$ & $(* *)$ & $\left({ }^{* *}\right)$ \\
\hline PropVap & & & $\left({ }^{* *}\right)$ & $(* *)$ & $\left({ }^{(*)}\right)$ & $\left({ }^{* *}\right)$ & $(* *)$ & $\left({ }^{(* *}\right)$ \\
\hline XSteam & & & $\left({ }^{* *}\right)$ & $(* *)$ & $\left({ }^{(*)}\right)$ & $\left({ }^{* *}\right)$ & $(* *)$ & $\left({ }^{* *}\right)$ \\
\hline
\end{tabular}

Table - 2: Results for the Saturation region

\begin{tabular}{|c|c|c|c|c|c|c|c|c|}
\hline \multicolumn{9}{|c|}{ Pressure and Enthalpy } \\
\hline Program & $\mathbf{P}$ & $\mathrm{H}$ & $\mathrm{T}$ & $\mathrm{s}$ & $\mathbf{v}$ & $\mathrm{U}$ & Ex & $\mathrm{x}$ \\
\hline This work & \multirow{4}{*}{100,0} & \multirow{4}{*}{2500,0} & 311,00 & 5,2295 & 0,0152 & 2348,07 & 945,27 & 0,8289 \\
\hline CATT 3 & & & 311,10 & 5,2290 & 0,0152 & 2348,00 & (*) & 0,8294 \\
\hline PropVap & & & 311,07 & 5,229 & 0,0152 & 2348,00 & 945,40 & 0,8293 \\
\hline XSteam & & & 311,00 & 5,2299 & 0,0152 & 2348,00 & $(*)$ & 0,8289 \\
\hline
\end{tabular}

\begin{tabular}{|c|c|c|c|c|c|c|c|c|}
\hline \multicolumn{9}{|c|}{ Pressure and Entropy } \\
\hline Program & $\mathbf{P}$ & $\mathrm{s}$ & $T$ & H & $\mathbf{v}$ & $\mathrm{U}$ & $\mathbf{E x}$ & $x$ \\
\hline This work & \multirow{4}{*}{120,0} & \multirow{4}{*}{5,0} & 324,68 & 2390,20 & 0,0111 & 2256,95 & 904,01 & 0,7529 \\
\hline CATT 3 & & & 324,70 & 2340,00 & 0,0111 & 2257,00 & $(*)$ & 0,7534 \\
\hline PropVap & & & 324,75 & 2390,40 & 0,0111 & 2257,00 & 904,25 & 0,7533 \\
\hline XSteam & & & 324,68 & 2390,20 & 0,0111 & 2256,80 & $\left({ }^{*}\right)$ & 0,7527 \\
\hline
\end{tabular}

\begin{tabular}{|c|c|c|c|c|c|c|c|c|}
\hline \multicolumn{9}{|c|}{ Pressure and Specific Volume } \\
\hline Program & $\mathbf{P}$ & $\mathbf{v}$ & $\mathrm{T}$ & $\mathbf{H}$ & $\mathrm{s}$ & $\mathrm{U}$ & Ex & $x$ \\
\hline This work & \multirow{4}{*}{210,0} & \multirow{4}{*}{0,004} & 369,83 & 2177,47 & 4,5573 & 2093,72 & 823,28 & 0,6514 \\
\hline CATT 3 & & & 369,90 & 2180,00 & 4,5600 & 2096,00 & $\left({ }^{*}\right)$ & 0,6531 \\
\hline PropVap & & & $\left({ }^{* *}\right)$ & $\left({ }^{* *}\right)$ & $\left({ }^{* *}\right)$ & $\left({ }^{* *}\right)$ & $\left({ }^{* *}\right)$ & $\left({ }^{(*)}\right)$ \\
\hline XSteam & & & $(* *)$ & $\left({ }^{* *}\right)$ & $(* *)$ & $(* *)$ & $(* *)$ & $\left({ }^{(*)}\right)$ \\
\hline
\end{tabular}

\begin{tabular}{|c|c|c|c|c|c|c|c|c|}
\hline \multicolumn{9}{|c|}{ Pressure and Internal Energy } \\
\hline Program & $\mathbf{P}$ & $\mathrm{U}$ & $\mathrm{T}$ & $\mathrm{H}$ & $\mathrm{s}$ & $\mathbf{v}$ & Ex & $x$ \\
\hline This work & \multirow{4}{*}{170,0} & \multirow{4}{*}{2100,00} & 352,29 & 2196,32 & 4,6173 & 0,0057 & 824,24 & 0,5902 \\
\hline CATT 3 & & & $(* *)$ & $(* *)$ & $(* *)$ & $(* *)$ & $(* *)$ & $\left({ }^{(* *)}\right.$ \\
\hline PropVap & & & $\left({ }^{* *}\right)$ & $\left({ }^{(* *)}\right.$ & $\left({ }^{* *}\right)$ & $\left({ }^{* *}\right)$ & $(* *)$ & $(* *)$ \\
\hline XSteam & & & $(* *)$ & $\left({ }^{(*)}\right)$ & $(* *)$ & $(* *)$ & $\left({ }^{* *}\right)$ & $\left({ }^{* *}\right)$ \\
\hline
\end{tabular}

\begin{tabular}{|c|c|c|c|c|c|c|c|c|}
\hline \multicolumn{9}{|c|}{ Pressure and Quality } \\
\hline Program & $P$ & $x$ & T & H & $\mathrm{S}$ & $\mathbf{v}$ & $\mathrm{U}$ & Ex \\
\hline This work & \multirow{4}{*}{120,0} & \multirow{4}{*}{0,40} & 324,68 & 1968,63 & 4,2948 & 0,0066 & 1889,52 & 692,71 \\
\hline CATT 3 & & & 324,70 & 1469,00 & 4,2950 & 0,0066 & 1889,00 & $(*)$ \\
\hline PropVap & & & 324,75 & \begin{tabular}{|l|}
1968,70 \\
\end{tabular} & 4,2947 & 0,0066 & 1889,30 & 692,82 \\
\hline XSteam & & & 324,68 & 1969,00 & $(*)$ & $\left({ }^{*}\right)$ & $\left({ }^{*}\right)$ & $\left({ }^{*}\right)$ \\
\hline
\end{tabular}

\begin{tabular}{|c|c|c|c|c|c|c|c|c|}
\hline \multicolumn{9}{|c|}{ Temperature and Enthalpy } \\
\hline Program & $\mathrm{T}$ & H & $\mathbf{P}$ & $\mathrm{S}$ & $v$ & $\mathrm{U}$ & Ex & $x$ \\
\hline This work & \multirow{4}{*}{230,0} & \multirow{4}{*}{2500,00} & 27,97 & 5,6108 & 0,0598 & 2332,86 & 831,70 & 0,8329 \\
\hline CATT 3 & & & $\left({ }^{(* *}\right)$ & $\left({ }^{* *}\right)$ & $\left({ }^{* *}\right)$ & $\left({ }^{* *}\right)$ & $\left({ }^{* *}\right)$ & ${ }^{(* *)}$ \\
\hline PropVap & & & $\left({ }^{* *}\right)$ & $\left({ }^{* *}\right)$ & $(* *)$ & $(* *)$ & $\left({ }^{* *}\right)$ & $\left({ }^{* *}\right)$ \\
\hline XSteam & & & $\left({ }^{* *}\right)$ & $\left({ }^{* *}\right)$ & $\left({ }^{(*)}\right)$ & $(* *)$ & $\left({ }^{* *}\right)$ & $\left({ }^{* *}\right)$ \\
\hline
\end{tabular}

\begin{tabular}{|c|c|c|c|c|c|c|c|c|}
\hline \multicolumn{9}{|c|}{ Temperature and Entropy } \\
\hline Program & $T$ & $\mathrm{~s}$ & $\mathbf{P}$ & H & $\mathbf{v}$ & $\mathrm{U}$ & $\mathbf{E x}$ & $x$ \\
\hline This work & \multirow{4}{*}{300,0} & \multirow{4}{*}{4,00} & 85,88 & 1771,95 & 0,0076 & 1707,45 & 583,91 & 0,3045 \\
\hline CATT 3 & & & 85,81 & 1772,00 & 0,0076 & 1707,00 & $\left({ }^{*}\right)$ & 0,3047 \\
\hline PropVap & & & $\left({ }^{* *}\right)$ & $\left({ }^{* *}\right)$ & $(* *)$ & $(* *)$ & $(* *)$ & $(* *)$ \\
\hline XSteam & & & $\left({ }^{* *}\right)$ & $\left({ }^{* *}\right)$ & $\left({ }^{* *}\right)$ & $(* *)$ & $(* *)$ & $\left({ }^{(* *)}\right)$ \\
\hline
\end{tabular}

\begin{tabular}{|c|c|c|c|c|c|c|c|c|}
\hline \multicolumn{9}{|c|}{ Temperature and Specific Volume } \\
\hline Program & $\mathbf{T}$ & $\mathbf{v}$ & $\mathbf{P}$ & $\mathbf{H}$ & $\mathrm{s}$ & $\mathrm{U}$ & Ex & $x$ \\
\hline This work & \multirow{4}{*}{40,0} & \multirow{4}{*}{2,00} & 0,07 & 414,00 & 1,3595 & 399,233 & 13,24 & 0,1024 \\
\hline CATT 3 & & & 0,07 & 414,00 & 1,3590 & 399,200 & $\left({ }^{*}\right)$ & 0,1024 \\
\hline PropVap & & & $(* *)$ & $(* *)$ & $(* *)$ & $(* *)$ & $\left({ }^{* *}\right)$ & $\left({ }^{(*)}\right)$ \\
\hline XSteam & & & $\left({ }^{(*)}\right)$ & $(* *)$ & $\left({ }^{(* 8)}\right.$ & $(* *)$ & $(* *)$ & $\left({ }^{(*)}\right)$ \\
\hline
\end{tabular}

\begin{tabular}{|c|c|c|c|c|c|c|c|c|}
\hline \multicolumn{9}{|c|}{ Temperature and Internal Energy } \\
\hline Program & $\mathrm{T}$ & $\mathrm{U}$ & $\mathbf{P}$ & $\mathbf{H}$ & $\mathrm{s}$ & $\mathbf{v}$ & Ex & $x$ \\
\hline This work & \multirow{4}{*}{190,0} & \multirow{4}{*}{1800,00} & 12,55 & 1910,15 & 4,6163 & 0,0877 & 538,35 & 0,5575 \\
\hline CATT 3 & & & $(* *)$ & ${ }^{(* *)}$ & $\left({ }^{* *}\right)$ & $(* *)$ & $\left({ }^{* *}\right)$ & $\left({ }^{(*)}\right)$ \\
\hline PropVap & & & $\left({ }^{* *}\right)$ & $\left({ }^{(*)}\right)$ & $\left({ }^{(*)}\right)$ & $(* *)$ & $(* *)$ & $\left({ }^{* *}\right)$ \\
\hline XSteam & & & $(* *)$ & $\left({ }^{(* 8)}\right.$ & $(* *)$ & $(* *)$ & $(* *)$ & $(* *)$ \\
\hline \multicolumn{9}{|c|}{ Temperature and Quality } \\
\hline Program & $\mathbf{T}$ & $\frac{x}{x}$ & $\mathbf{P}$ & $\mathbf{H}$ & $\mathrm{s}$ & $\mathbf{v}$ & $\mathrm{U}$ & $\mathbf{E x}$ \\
\hline This work & \multirow{4}{*}{40,0} & \multirow{4}{*}{0,70} & 0,07 & 1851,71 & 5,9506 & 13,6608 & 1750,84 & 82,09 \\
\hline CATT 3 & & & 0,07 & 1852,00 & 5,9520 & 13,6700 & 1751,00 & $(*)$ \\
\hline PropVap & & & $(* *)$ & $(* *)$ & $(* *)$ & $(* *)$ & $(* *)$ & $(* *)$ \\
\hline XSteam & & & 0,07 & 1851,70 & $(*)$ & $(*)$ & $\left({ }^{*}\right)$ & $(*)$ \\
\hline
\end{tabular}

Table 3 - Results for the Compressed Liquid region

\begin{tabular}{|c|c|c|c|c|c|c|c|c|}
\hline \multicolumn{9}{|c|}{ Pressure and Temperature } \\
\hline Program & $\mathbf{P}$ & $\mathrm{T}$ & H & $\mathrm{s}$ & $\mathbf{v}$ & $\mathrm{U}$ & Ex & $x$ \\
\hline This work & \multirow{4}{*}{40,0} & \multirow{4}{*}{100,0} & 422,10 & 1,3042 & 0,0010 & 417,937 & 37,82 & $-0,4560$ \\
\hline CATT 3 & & & 422,00 & 1,3040 & 0,0010 & 417,800 & $\left({ }^{*}\right)$ & $\left({ }^{*}\right)$ \\
\hline PropVap & & & 421,97 & 1,3038 & 0,0010 & 417,800 & 37,80 & $-0,3882$ \\
\hline XSteam & & & 422,03 & 1,3040 & 0,0010 & 417,864 & $\left({ }^{*}\right)$ & $\left({ }^{*}\right)$ \\
\hline \multicolumn{9}{|c|}{ Pressure and Enthalpy } \\
\hline Program & $\mathbf{P}$ & $\mathbf{H}$ & $\mathrm{T}$ & $\mathrm{s}$ & $\mathbf{v}$ & $\mathrm{U}$ & Ex & $x$ \\
\hline This work & & \multirow{4}{*}{1200,0} & 273,18 & 2,9928 & 0,0013 & 1186,97 & 312,26 & $-0,1629$ \\
\hline CATT 3 & & & 273 & 2,9930 & 0,0013 & 1187,00 & $(*)$ & $(*)$ \\
\hline PropVap & & & 273,34 & 2,9927 & 0,0013 & 1187,00 & 312,28 & $-1,5759$ \\
\hline XSteam & & & 273,21 & 2,9927 & 0,0013 & 1186,90 & $(*)$ & $\left({ }^{*}\right)$ \\
\hline
\end{tabular}

\begin{tabular}{|c|c|c|c|c|c|c|c|c|}
\hline \multicolumn{9}{|c|}{ Pressure and Entropy } \\
\hline Program & $\mathbf{P}$ & $\mathrm{s}$ & $T$ & H & $\mathbf{v}$ & $\mathrm{U}$ & $\mathbf{E x}$ & $x$ \\
\hline This wo & \multirow{4}{*}{180,0} & \multirow{4}{*}{3,0} & 284,47 & 1224,58 & 0,0013 & 1201,16 & 334,69 & $-0,7064$ \\
\hline CATT 3 & & & 276,60 & 1214,00 & 0,0012 & 1191,00 & $\left.{ }^{*}{ }^{*}\right)$ & $\left({ }^{*}\right)$ \\
\hline PropVap & & & 276,63 & 1214,40 & 0,0013 & 1191,10 & 324,47 & $-0,6661$ \\
\hline XStea & & & 276,50 & 1214,30 & 0,0013 & 1191,10 & $(*)$ & $(*)$ \\
\hline
\end{tabular}

\begin{tabular}{|c|c|c|c|c|c|c|c|c|}
\hline \multicolumn{9}{|c|}{ Pressure and Specific Volume } \\
\hline Program & $\mathbf{P}$ & $\mathbf{v}$ & $T$ & $\mathrm{H}$ & $\mathrm{s}$ & $\mathrm{U}$ & Ex & $x$ \\
\hline This work & \multirow{4}{*}{260,0} & \multirow{4}{*}{0,0011} & 173,53 & 748,48 & 2,0450 & 719,882 & 143,33 & $-0,0065$ \\
\hline CATT 3 & & & 173,70 & 749,30 & 2,0470 & 720,700 & $(*)$ & $\left({ }^{*}\right)$ \\
\hline PropVap & & & $(* *)$ & $\left({ }^{(*)}\right)$ & $(* *)$ & $(* *)$ & $\left({ }^{* *}\right)$ & $\left({ }^{* *}\right)$ \\
\hline XSteam & & & $(* *)$ & $(* *)$ & $(* *)$ & $(* *)$ & ${ }^{(* *)}$ & $(* *)$ \\
\hline
\end{tabular}

\begin{tabular}{|c|c|c|c|c|c|c|c|c|}
\hline \multicolumn{9}{|c|}{ Pressure and Internal Energy } \\
\hline Program & $P$ & $\mathrm{U}$ & $\mathrm{T}$ & H & $\mathrm{s}$ & $\mathbf{v}$ & Ex & $x$ \\
\hline This work & \multirow{4}{*}{150,0} & \multirow{4}{*}{1000,00} & 235,44 & 1018,05 & 2,6360 & 0,0012 & 236,68 & \begin{tabular}{|l|l|}
$-0,6450$ \\
\end{tabular} \\
\hline CATT 3 & & & $\left({ }^{* *}\right)$ & ${ }^{(* *)}$ & $(* *)$ & $\left({ }^{* *}\right)$ & ${ }^{(* *)}$ & $\left({ }^{* *}\right)$ \\
\hline PropVap & & & $(* *)$ & $\left({ }^{(* *)}\right)$ & $\left({ }^{(* *}\right)$ & $\left({ }^{* *}\right)$ & ${ }^{(* *)}$ & $\left({ }^{* *}\right)$ \\
\hline XSteam & & & $\left({ }^{* *}\right)$ & $\left({ }^{(* *}\right)$ & $\left({ }^{* *}\right)$ & $\left({ }^{* *}\right)$ & ${ }^{(* *)}$ & $\left({ }^{(*)}\right)$ \\
\hline
\end{tabular}

\begin{tabular}{|c|c|c|c|c|c|c|c|c|}
\hline \multicolumn{9}{|c|}{ Temperature and Entropy } \\
\hline Program & $\mathrm{T}$ & $\mathrm{s}$ & $\mathbf{P}$ & H & $\mathbf{v}$ & $\mathrm{U}$ & $\mathbf{E x}$ & $x$ \\
\hline This work & \multirow{4}{*}{300,0} & \multirow{4}{*}{3,10} & 93,72 & 1323,51 & 0,001 & 1248,04 & 403,81 & $-0,0625$ \\
\hline CATT 3 & & & 586,20 & 1323,00 & 0,001 & 1248,00 & $\left({ }^{*}\right)$ & $\left({ }^{*}\right)$ \\
\hline PropVap & & & $(* *)$ & $\left({ }^{* *}\right)$ & $(* *)$ & $\left({ }^{* *}\right)$ & $\left({ }^{(* *)}\right.$ & $(* *)$ \\
\hline XSteam & & & $(* *)$ & $(* *)$ & $(* *)$ & $(* *)$ & $\left({ }^{(*)}\right)$ & $(* *)$ \\
\hline
\end{tabular}




\begin{tabular}{|c|c|c|c|c|c|c|c|c|}
\hline \multicolumn{9}{|c|}{ Temperature and Specific Volume } \\
\hline Program & T & $\mathbf{v}$ & $\mathbf{P}$ & $\mathrm{H}$ & s & $\mathrm{U}$ & $\mathbf{E x}$ & $x$ \\
\hline This work & \multirow{4}{*}{290,0} & \multirow{4}{*}{0,0013} & 314,91 & 1278,39 & 3,0838 & 1237,45 & 363,52 & $-0,0288$ \\
\hline CATT 3 & & & 306,80 & 1278,00 & 3,0840 & 1238,00 & $(*)$ & $\left.{ }^{*}\right)$ \\
\hline PropVap & & & $(* *)$ & $\left({ }^{(*)}\right)$ & $(* *)$ & $(* *)$ & $\left({ }^{* *}\right)$ & $(* *)$ \\
\hline XSte & & & $(* *)$ & $\left({ }^{* *}\right)$ & $\left({ }^{* *}\right)$ & $(* *)$ & $\left({ }^{* *}\right)$ & $\left({ }^{* *}\right)$ \\
\hline
\end{tabular}

\begin{tabular}{|c|c|c|c|c|c|c|c|c|}
\hline \multicolumn{9}{|c|}{ Temperature and Internal Energy } \\
\hline Program & $T$ & $\mathrm{U}$ & $P$ & $\mathrm{H}$ & $\mathrm{s}$ & $\mathbf{v}$ & Ex & $x$ \\
\hline This work & \multirow{4}{*}{200,0} & \multirow{4}{*}{820,00} & 490,04 & 874,67 & 2,2641 & 0,0011 & 204,20 & $-0,0163$ \\
\hline CATT 3 & & & $(* *)$ & $(* *)$ & $(* *)$ & $(* *)$ & $\left({ }^{(* *}\right)$ & $(* *)$ \\
\hline PropVap & & & $(* *)$ & $\left({ }^{(* *}\right)$ & $(* *)$ & $(* *)$ & $\left({ }^{(* *)}\right.$ & $\left({ }^{(* *}\right)$ \\
\hline XSteam & & & $(* *)$ & $\left({ }^{(* *)}\right.$ & $(* *)$ & $\left({ }^{* *}\right)$ & $\left({ }^{(* *)}\right.$ & $\left({ }^{(* *}\right)$ \\
\hline
\end{tabular}

Comparing the results, especially when one of the options contain the pressure, the results for the thermodynamic properties seem to be very similar, the exception of quality. The qualities obtained in this work and the PropVap software are very different for the cases of $\mathrm{P}$ and $\mathrm{T}, \mathrm{P}$ and $\mathrm{H}$ and $\mathrm{P}$ and $\mathrm{S}$. For the quality, the criterion used in this work is:

$x=\frac{H_{(P, T)}-H_{(P)}^{L S}}{H_{(P)}^{V S}-H_{(P)}^{L S}}$

where $\mathrm{x}$ is the quality, $\mathrm{H}$ is the enthalpy $(\mathrm{kJ} / \mathrm{kg})$, LS and VS are the saturated liquid and saturated vapor, $\mathrm{P}$ is the pressure and $\mathrm{T}$ is the temperature.

To explain these differences in the results, it can be used the option $\mathrm{T}$ and $\mathrm{H}$ in the Compressed Liquid region. The tendency of the thermodynamic properties is decrease with the increase of the pressure and increase with the increase of the temperature. However, the behavior of the enthalpy in the Compressed Liquid region is different. This property increase with the increase of the temperature and increase with the increase of the pressure too, instead of other thermodynamic properties, which have a normal behavior. This behavior causes an abnormality. It is the fact that all values of enthalpy in Compressed Liquid region are also very close to the Saturation region. When the compressed liquid and saturated liquid are very close, the Computer program developed in this work warns about this behavior to user, showing the sentence "Under these conditions, the system also may be Compressed Liquid". Due to that enthalpies of compressed liquid and enthalpies of the saturated liquid are very similar, the quality in these cases could be calculated with other thermodynamic property, such as entropy.

Another discussion happens, in Table 3, for the option P and $\mathrm{H}$ in the Compressed Liquid region. Differences between the values of quality is larger for results obtained in this work and the program PropVap. By using the program CATT 3, which provides a phase diagram of water, it is shown the thermodynamic region and the point in the region where the thermodynamic state is located. It is possible to observe, that input values, that is: $\mathrm{P}=100,0$ bar and $\mathrm{H}=1200,0 \mathrm{~kJ} / \mathrm{kg}$, that the thermodynamic state is located near to the frontier between Compressed Liquid and the region of saturated liquid. With this localization, it is possible conclude that the value of quality near of $0,0(-0,1629)$, calculated in the program of this work) is more exact than the value of quality calculated for PropVap $(-1,5759)$, showing a water like compressed liquid very distant from Saturated region. This reveal that the calculation method used in the program of this work is more accurate and, based on the results obtained too, provides results with a good accuracy and reliability.

Table 4 presents the results obtained in this work and the results obtained with the other three programs in the supercritical region. When two thermodynamic propertiesare given, that is: $\mathrm{P}$ and $\mathrm{T}, \mathrm{P}$ and $\mathrm{H}, \mathrm{P}$, and $\mathrm{S}, \mathrm{P}$ and $\mathrm{v}, \mathrm{P}$ and $\mathrm{U}, \mathrm{T}$ and $\mathrm{H}, \mathrm{T}$ and $\mathrm{S}, \mathrm{T}$ and $\mathrm{v}$ and $\mathrm{T}$ and $\mathrm{U}$, the other thermodynamic properties are calculated. In this table, some thermodynamic properties have not been implemented for the option chosen $(*)$ and many results were not found for the programs because the chosen options were not implemented (**).

Table - 4: Results for the Supercritical region

\begin{tabular}{|c|c|c|c|c|c|c|c|c|}
\hline \multicolumn{9}{|c|}{ Pressure and Temperature } \\
\hline Program & $\mathbf{P}$ & $\mathrm{T}$ & $\mathbf{H}$ & $\mathrm{S}$ & $\mathbf{v}$ & $\mathrm{U}$ & Ex & $x$ \\
\hline This work & \multirow{4}{*}{500,0} & \multirow{4}{*}{400,0} & 1874,40 & 4,0029 & 0,0017 & 1787,8 & 685,50 & Indefinite \\
\hline CATT 3 & & & 1875,00 & 4,0030 & 0,0017 & 1788,0 & $\left({ }^{*}\right)$ & $\left({ }^{*}\right)$ \\
\hline PropVap & & & $(* *)$ & $\left({ }^{* *}\right)$ & $\left({ }^{* *}\right)$ & $\left({ }^{* *}\right)$ & $\left({ }^{* *}\right)$ & $\left({ }^{* *}\right)$ \\
\hline XSteam & & & 1874,40 & 4,0029 & 0,0017 & 1787,8 & $\left({ }^{*}\right)$ & $\left({ }^{*}\right)$ \\
\hline
\end{tabular}

\begin{tabular}{|c|c|c|c|c|c|c|c|c|}
\hline \multicolumn{9}{|c|}{ Pressure and Enthalpy } \\
\hline Program & $\mathbf{P}$ & $\mathrm{H}$ & $T$ & $\mathrm{~s}$ & $\mathbf{v}$ & $\mathrm{U}$ & $E x$ & $\bar{x}$ \\
\hline This work & \multirow{4}{*}{725,0} & \multirow{4}{*}{2800,0} & 554,75 & 5,1690 & 0,0032 & 2567,00 & 1263,41 & Indefinite \\
\hline CATT 3 & & & 555,60 & 5,1740 & 0,0032 & 2570,00 & $(*)$ & $(*)$ \\
\hline PropVap & & & $\left({ }^{* *}\right)$ & ${ }^{(* *)}$ & ${ }^{(* *)}$ & $\left({ }^{* *}\right)$ & $\left({ }^{* *}\right)$ & $\left({ }^{* *}\right)$ \\
\hline XSteam & & & 554,74 & 5,1750 & 0,0032 & 2571,20 & $\left({ }^{*}\right)$ & $(*)$ \\
\hline
\end{tabular}

\begin{tabular}{|c|c|c|c|c|c|c|c|c|}
\hline \multicolumn{9}{|c|}{ Pressure and Entropy } \\
\hline Progra & $P$ & - & $T$ & H & $\mathbf{v}$ & $\mathrm{U}$ & $E x$ & $x$ \\
\hline This work & \multirow{4}{*}{400,0} & \multirow{4}{*}{7,0} & 938,63 & 4359,08 & 0,0135 & 3818,22 & 2276,59 & Indefinite \\
\hline CATT 3 & & & 937,30 & 4359,00 & 0,0013 & 3820,00 & $(*)$ & $(*)$ \\
\hline PropVap & & & $(* *)$ & $(* *)$ & $\left({ }^{* *}\right)$ & $\left({ }^{* *}\right)$ & $(* *)$ & $(* *)$ \\
\hline XSteam & & & $\left({ }^{* *}\right)$ & $\left({ }^{* *}\right)$ & $\left({ }^{* *}\right)$ & $\left({ }^{(*)}\right)$ & $\left({ }^{* *}\right)$ & $\left({ }^{* *}\right)$ \\
\hline
\end{tabular}

\begin{tabular}{|c|c|c|c|c|c|c|c|c|}
\hline \multicolumn{9}{|c|}{ Pressure and Specific Volume } \\
\hline Program & $\mathbf{P}$ & $\mathbf{v}$ & $\mathrm{T}$ & H & $\mathrm{s}$ & $\mathrm{U}$ & Ex & 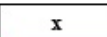 \\
\hline This work & \multirow{4}{*}{310,0} & \multirow{4}{*}{0,01} & 540,03 & 3202,89 & 5,9291 & 2914,64 & 1439,71 & Indefinite \\
\hline CATT 3 & & & 559,20 & 3296,00 & 6,0470 & 2986,00 & $\left({ }^{*}\right)$ & $\left({ }^{*}\right)$ \\
\hline PropVap & & & $(* *)$ & $(* *)$ & $(* *)$ & $\left({ }^{* *}\right)$ & $\left({ }^{* *}\right)$ & $\left({ }^{(*)}\right)$ \\
\hline XSteam & & & $(* *)$ & $(* *)$ & $(* *)$ & $\left({ }^{(* *)}\right.$ & $\left({ }^{(* *)}\right.$ & $\left({ }^{(* *)}\right.$ \\
\hline
\end{tabular}

\begin{tabular}{|c|c|c|c|c|c|c|c|c|}
\hline \multicolumn{9}{|c|}{ Pressure and Internal Energy } \\
\hline Program & $P$ & $\mathrm{U}$ & $\mathrm{T}$ & $\mathrm{H}$ & $\mathrm{s}$ & $\mathbf{v}$ & $E x$ & $x$ \\
\hline This work & \multirow{4}{*}{350,0} & \multirow{4}{*}{2700,00} & 486,87 & 2937,57 & 5,5569 & 0,0065 & 1285,35 & Indefinite \\
\hline CATT 3 & & & $\left({ }^{* *}\right)$ & $\left({ }^{* *}\right)$ & $(* *)$ & $\left({ }^{* *}\right)$ & $\left({ }^{* *}\right)$ & $(* *)$ \\
\hline PropVap & & & $(* *)$ & $(* *)$ & $(* *)$ & $(* *)$ & $\left({ }^{* *}\right)$ & $(* *)$ \\
\hline XSteam & & & $(* *)$ & $(* *)$ & $(* *)$ & $\left({ }^{* *}\right)$ & $(* *)$ & $(* 8)$ \\
\hline
\end{tabular}

\begin{tabular}{|c|c|c|c|c|c|c|c|c|}
\hline \multicolumn{9}{|c|}{ Temperature and Enthalpy } \\
\hline Program & $\mathrm{T}$ & $\mathrm{H}$ & $\mathbf{P}$ & $\mathrm{s}$ & $\mathbf{v}$ & $\mathrm{U}$ & Ex & $x$ \\
\hline This work & \multirow{4}{*}{600,0} & \multirow{4}{*}{3500,00} & 242,92 & 6,3833 & 0,0146 & 3145,06 & 1601,38 & Indefinite \\
\hline CATT 3 & & & $\left({ }^{(* *}\right)$ & $(* *)$ & $(* *)$ & $\left({ }^{(* *}\right)$ & $\left({ }^{* *}\right)$ & $\left({ }^{* *}\right)$ \\
\hline PropVap & & & $(* *)$ & $\left({ }^{* 8}\right)$ & $(* *)$ & $\left({ }^{* *}\right)$ & $\left({ }^{* *}\right)$ & $\left({ }^{(* *)}\right)$ \\
\hline XSteam & & & $(* *)$ & $\left({ }^{* *}\right)$ & $(* *)$ & $\left({ }^{* *}\right)$ & $\left({ }^{(*)}\right)$ & $\left({ }^{(*)}\right)$ \\
\hline
\end{tabular}




\begin{tabular}{|c|c|c|c|c|c|c|c|c|}
\hline \multicolumn{9}{|c|}{ Temperature and Entropy } \\
\hline Program & $T$ & $\mathrm{~S}$ & $\mathbf{P}$ & $\mathbf{H}$ & $\mathbf{v}$ & $\mathrm{U}$ & $\mathbf{E x}$ & $x$ \\
\hline This work & \multirow{4}{*}{400,0} & \multirow{4}{*}{5,00} & 263,60 & 2472,62 & 0,0051 & 2339,17 & 986,43 & Indefinite \\
\hline CATT 3 & & & 262,50 & 2492,00 & 0,005100 & 2358,00 & $(*)$ & $(*)$ \\
\hline PropVap & & & $\left({ }^{* *}\right)$ & $\left({ }^{* *}\right)$ & $\left({ }^{* *}\right)$ & $\left({ }^{* *}\right)$ & $\left({ }^{* *}\right)$ & $(* *)$ \\
\hline XSteam & & & $(* *)$ & $\left({ }^{* *}\right)$ & $(* *)$ & $(* *)$ & $(* *)$ & $(* *)$ \\
\hline
\end{tabular}

\begin{tabular}{|c|c|c|c|c|c|c|c|c|}
\hline \multicolumn{9}{|c|}{ Temperature and Specific Volume } \\
\hline Program & $T$ & $\mathbf{v}$ & $\mathbf{P}$ & $\mathbf{H}$ & $\mathbf{S}$ & $\mathrm{U}$ & $\mathbf{E x}$ & $x$ \\
\hline This work & \multirow{4}{*}{500,0} & \multirow{4}{*}{0,01} & 273,35 & 3128,03 & 5,8855 & 2857,74 & 1377,82 & Indefinite \\
\hline CATT 3 & & & 270,80 & 3129,00 & 5,8880 & 2858,00 & $(*)$ & $(*)$ \\
\hline PropVap & & & $\left({ }^{* *}\right)$ & $(* *)$ & $(* *)$ & $(* *)$ & $(* *)$ & $(* *)$ \\
\hline XSteam & & & $\left({ }^{* *}\right)$ & $\left({ }^{* *}\right)$ & $(* *)$ & $(* *)$ & $(* *)$ & $(* *)$ \\
\hline
\end{tabular}

\begin{tabular}{|c|c|c|c|c|c|c|c|c|}
\hline \multicolumn{9}{|c|}{ Temperature and Internal Energy } \\
\hline Program & $T$ & $\mathrm{U}$ & $\mathbf{P}$ & $\mathbf{H}$ & $\mathrm{S}$ & $\mathbf{v}$ & Ex & $x$ \\
\hline This work & \multirow{4}{*}{450,0} & \multirow{4}{*}{2200,00} & 498,84 & 2327,56 & 4,6883 & 0,0026 & 934,31 & Indefinite \\
\hline CATT 3 & & & $(* *)$ & $\left({ }^{(* *)}\right.$ & $\left({ }^{* *}\right)$ & $(* *)$ & $\left({ }^{* *}\right)$ & $(* *)$ \\
\hline PropVap & & & $(* *)$ & $(* *)$ & $(* *)$ & $\left({ }^{* *}\right)$ & $\left({ }^{* *}\right)$ & $(* *)$ \\
\hline XSteam & & & $(* *)$ & $(* *)$ & $(* *)$ & $(* *)$ & $\left({ }^{* *}\right)$ & $(* *)$ \\
\hline
\end{tabular}

Computational programs: CATT 3, ProVap and Xsteamare not implemented for the following cases: $\mathrm{P}$ and $\mathrm{U}, \mathrm{T}$ and $\mathrm{H} \mathrm{e}$ $\mathrm{T}$ and $\mathrm{U}$. When the comparison is done between the results obtained in this work with the others ones, especially when the option contains $\mathrm{P}$ and $\mathrm{T}$ and $\mathrm{P}$ and $\mathrm{H}$, the results obtained in this work for the thermodynamic properties seem also to be very similar with the ones obtained with the CATT 3, and Xsteam programs. The same can be said when the temperature is included in one of the options chosen by the user ( $\mathrm{T}$ and $\mathrm{S}$ ). For the case of $\mathrm{P}$ and $\mathrm{S}, \mathrm{P}$ and $\mathrm{v}$, the results obtained in this work and CATT 3 are very similar.

Quality has not been calculated for the supercritical region because for the supercritical pressures and temperatures there are not enthalpies of the saturated liquid $\left(\mathrm{H}_{(\mathrm{P})}^{\mathrm{VS}}\right)$ and saturated vapor $\left(\mathrm{H}_{(\mathrm{P})}^{\mathrm{LS}}\right)$. So the quality for the fluid in the supercritical region was written as "indefinite".

In supercritical region, errors raised due to severe conditions of pressure and temperature which water is submitted, causing a difference between the results obtained with the programs. Besides, some errors are caused because the spline models used by the MatLab are very sensitives, causing a little difference in high values of temperatures and pressures.

As noted in the tables, the program developed in this work has more options than other programs and has a bigger working range, besides it provides more properties, such as exergy. Results that are presented by all programs through the values of thermodynamic properties are very near, with a low relative error between them.

These errors are originated, mainly, from the different reference states used in the construction of the programs. Authors, for the construction of thermodynamic tables, get different values of thermodynamic properties of fluid due to the different conditions and reference states.

Programs used in this workcan be downloaded from the following links:

- For users of MatLab:

https://www.dropbox.com/s/1xyzipmizvx7x0k/Thermo _web.rar?dl=0
- $\quad$ For non-users of Matlab:

https:/www.dropbox.com/s/13bqxff96anoaom/Thermo mor.rar? $\mathrm{dl}=0$

\section{CONCLUSION}

From results and the small errors between them, it is possible conclude that the program developed in this work presents a good accuracy to predict thermodynamic properties of water, comparable with other engineering programs available. The advantage of this program is the large variety of options (eleven) to calculate the thermodynamic properties of water and the large working range (above the critical point), larger than others programs (PropVap, CATT 3 and Xsteam), in addition for predicting in the supercritical fluidregion. Thus, this program represents a useful tool for teaching in courses of Applied Thermodynamics and can help in the calculation and design of equipments in industry.

\section{ACKNOWLEDGEMENT}

N.V. Freire thanks the Chemical Engineering Department of the Engineering School of Lorena, University of São Paulo (USP) for the opportunity given to him to get his scientific initiation and P.F. Arce-Castillo thanks the "Fundação de Amparo à Pesquisa do Estado de São Paulo (FAPESP)" the financial aid through the research grant: 2015/05155-8.

\section{REFERENCES}

[1]. Çengel, Y.A., Boles, M.A., Termodinâmica. McGraw Hill Publishers, Porto Alegre, Brazil, 2013.

[2]. Çengel Y.A., Ghajar A.J. Transferência de Calor e Massa. McGraw Hill Publishers, 4th Ed. in portuguese, Porto Alegre, Brazil, 2012.

[3]. Borgnakke C., Sonntag R.E. Fundamentos da Termodinâmica, BlucherPublishers, São Paulo, Brazil, 2013.

[4]. Kroos K.A., Potter M.C. Termodinâmica para Engenheiros. Cengage Learning Publishers, 1st Ed. in portuguese, Brazil, 2015.

[5]. Singh O. Applied Thermodynamics. New Age International Limited Publishers, 3th Ed., 2009.

[6]. Granet, I., Termodinâmica e Energia Térmica.PrenticeHall Publishers, 4th Ed., Rio de Janeiro, Brazil, 1990.

[7]. Walas, S.M., Chemical Process Equipment - Selection and Design. Butterworth-Heinemann Series in Chemical Engineering, Newton, MA, EUA, 1990.

[8]. Shvets I., Tolubinsky V., Kirakovsky N., Neduzhy I., Sheludko I. Heat engineering. Mir Publishers, 2nd Ed., Moscow, 1980.

[9]. Michelsen, M.L., Mollerup, J.M., Thermodynamic Models: Fundamentals \& Computational Aspects. Tie Line Publications, 2nd Ed., Denmark, 2007.

[10]. PROPVAP.J.Llagostera, Faculty of Mechanical Engineering, University of Campinas, v. 3.03, 1998.

[11]. Computer-Aided Thermodynamic Tables (CATT), v. 3.John Wiley \& Sons, Inc., 1996. 